\title{
Chemoembolization of Hepatocellular Carcinoma
}

\author{
Riccardo Lencioni, MD, FSIR, EBIR ${ }^{1}$ Pasquale Petruzzi, MD ${ }^{1} \quad$ Laura Crocetti, MD, PhD, EBIR ${ }^{1}$ \\ ${ }^{1}$ Division of Diagnostic Imaging and Intervention, Pisa University \\ School of Medicine, Pisa, Italy \\ Semin Intervent Radiol 2013;30:3-11

\begin{abstract}
Address for correspondence Riccardo Lencioni, MD, FSIR, EBIR, Diagnostic Imaging and Intervention, Pisa University School of Medicine, Building No. 29, 2nd floor, Via Paradisa 2, IT-56124 Pisa, Italy (e-mail: riccardo.lencioni@med.unipi.it).
\end{abstract}

\begin{abstract}
Keywords

- chemoembolization

- hepatocellular carcinoma

- transcatheter therapy

Transarterial chemoembolization (TACE) is the current standard of care for patients with intermediate-stage hepatocellular carcinoma (HCC) and relatively preserved liver function. In a meta-analysis of randomized controlled trials comparing conventional TACE regimens-including the administration of an anticancer-in-oil emulsion followed by embolic agents-versus best supportive care, TACE was shown to improve median survival from 16 to 20 months. Various strategies to improve outcomes for this patient group have become the subject of much ongoing clinical research. The introduction of an embolic drug-eluting bead (DEB) has been shown to substantially improve the pharmacokinetic profile of TACE, providing levels of consistency and repeatability not available with conventional regimens while concomitantly significantly diminishing systemic drug exposure. In randomized trials, DEB-TACE significantly reduced liver toxicity and drug-related adverse events compared with conventional TACE. In this article, technique, indications and contraindications, and clinical outcomes of conventional and DEB-TACE in the management of HCC are reviewed. In addition, scientific background and early clinical experience with the use of combination regimens including TACE and systemically active molecular-targeted agents with antiangiogenic properties are discussed. The combination of DEB-TACE and antiangiogenic therapy represents a potentially powerful approach that is currently undergoing clinical investigation in a phase 3 setting.
\end{abstract}

Objectives: Upon completion of this article, the reader will be able to discuss the role of chemoembolization in the treatment of hepatocellular carcinoma, including the outcomes of recent major studies as well as the concept of combination therapies.

Accreditation: This activity has been planned and implemented in accordance with the Essential Areas and policies of the Accreditation Council for Continuing Medical Education through the joint sponsorship of Tufts University School of Medicine (TUSM) and Thieme Medical Publishers, New York. TUSM is accredited by the ACCME to provide continuing medical education for physicians.

Credit: Tufts University School of Medicine designates this journal-based CME activity for a maximum of 1 AMA PRA Category 1 Credit $^{\mathrm{TM}}$. Physicians should claim only the credit commensurate with the extent of their participation in the activity.
Hepatocellular carcinoma (HCC) is the sixth most common cancer and the third leading cause of cancer-related death worldwide. ${ }^{1}$ Unlike most solid cancers, future incidence and mortality rates for HCC are projected to largely increase in several regions around the world over the next 20 years, mostly as a result of the dissemination of hepatitis $C$ virus infection. ${ }^{2,3}$ Despite the widespread implementation of surveillance programs of at-risk populations, most patients with HCC are diagnosed late when curative treatments cannot be applied. ${ }^{4}$ In addition, in a high proportion of cases the disease recurs after attempts at curative therapy. ${ }^{4}$

Transcatheter arterial chemoembolization (TACE) is the current standard of care for patients with large or multinodular HCC and relatively preserved liver function, absence of cancer-related symptoms, and no evidence of vascular invasion or extrahepatic spread (i.e., those classified as
Issue Theme Liver Malignancies; Guest Editor, Daniel B. Brown, MD, FSIR
Copyright (c) 2013 by Thieme Medical Publishers, Inc., 333 Seventh Avenue, New York, NY 10001, USA. Tel: +1(212) 584-4662.
Dol http://dx.doi.org/ $10.1055 / \mathrm{s}-0033-1333648$. ISSN 0739-9529. 


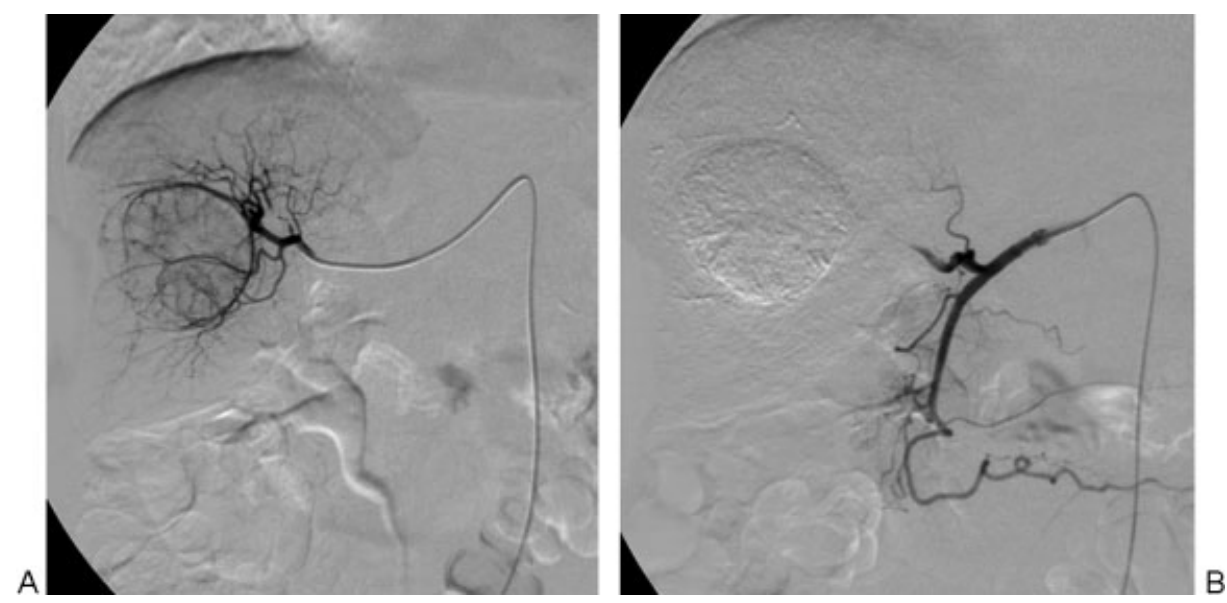

Figure 1 Conventional transcatheter arterial chemoembolization. (A) Following superselective catheterization of the hepatic arterial branch feeding the tumor, doxorubicin-in-oil emulsion is administered, followed by gelatin sponge particles. (B) Angiographic image obtained after the procedure shows absence of residual tumor vascularity.

intermediate stage according to the Barcelona Clinic Liver Cancer (BCLC) staging system). ${ }^{5-7}$ Although the administration of an anticancer-in-oil emulsion followed by embolic agents has been the most popular TACE technique, the introduction of an embolic drug-eluting bead (DEB) has provided an attractive alternative to conventional regimens. ${ }^{8}$ Clinical studies have shown that DEB loaded with doxorubicin has a safe pharmacokinetic profile with lower systemic drug exposure and significantly reduced liver toxicity compared with conventional TACE. ${ }^{9-11}$

Despite these advances and technical refinements, the long-term survival of patients managed with TACE are not fully satisfactory, mainly as a result of the high rates of tumor recurrence. TACE exerts therapeutic effects only in the treated territory; thus other HCC undetected at the time of the procedure may progress or new tumors may develop. Moreover, by interrupting blood flow to the tumor, TACE induces necrosis at the site of disease but may create conditions that permit or even encourage angiogenesis. ${ }^{12}$ Surrogate markers of tissue hypoxia that increase after TACE include hypoxia inducible factor $1 \alpha$ and both plasma and hepatic vascular endothelial growth factor (VEGF). Thus the combination of TACE with antiangiogenic agents is appealing because the systemically active drug might curtail the post-TACE rise in VEGF-mediated signaling and at the same time target any tumor foci distant from the site of treatment. ${ }^{12}$

In this article, technique, indications and contraindications, and clinical outcomes of conventional and DEB-TACE in the management of HCC are reviewed. In addition, early clinical experiences with the combination of TACE and antiangiogenic therapies are discussed.

\section{Technique}

The rationale for conventional TACE is that the intra-arterial injection of a viscous emulsion, made by a chemotherapeutic drug such as doxorubicin or cisplatin mixed with iodized oil, followed by embolization of the blood vessel with gelatin sponge particles or other embolic agents, will result in a strong cytotoxic effect enhanced by ischemia ${ }^{13}$ (-Fig. 1). An important limitation of conventional TACE has been the inconsistency in the technique and the treatment schedules. This limitation has hampered the acceptance of TACE as a standard oncologic treatment. DEB-TACE provides levels of consistency and repeatability not available with conventional TACE, and it offers the opportunity to implement a more standardized approach to HCC treatment. ${ }^{14}$

\section{Pretreatment Imaging}

Obtaining triple-phase computed tomography (CT) or magnetic resonance imaging (MRI) of the liver is mandatory to integrate clinical and laboratory data in evaluating the appropriateness of TACE by the local multidisciplinary liver tumor board. Additional imaging examinations to rule out extrahepatic disease should be performed as appropriate.

\section{Periprocedural Medication}

Pain medication should be given according to standard hospital protocols. Antibiotic prophylaxis and gastric protection should be administered at the physician's discretion.

\section{Choice and Dose of Chemotherapeutic Agent}

There is no consensus on the optimal chemotherapeutic agent(s) to use in conventional TACE. Worldwide, the most popular anticancer drug for TACE of HCC is doxorubicin. In conventional TACE, the dose of doxorubicin typically ranges from 30 to $75 / \mathrm{m}^{2}$, to a maximum of $150 \mathrm{mg}$. The drug is usually mixed with 5 to $20 \mathrm{~mL}$ of lipiodol. In DEB-TACE, different approaches have been recommended for patients with limited disease (defined as HCC within the Milan criteria for liver transplantation: single tumor $\leq 5 \mathrm{~cm}$, or multiple tumors (up to three, $\leq 3 \mathrm{~cm}$ each), or more advanced disease. ${ }^{14}$ These distinct populations are as follows:

1. Patients within the Milan criteria: As a general rule, each single treatment should include a planned dose of 50 to $75 \mathrm{mg}$ doxorubicin loaded into one vial containing $2 \mathrm{~mL}$ of 
DC Beads (loading dose, 25 to $37.5 \mathrm{mg}$ doxorubicin/mL of beads).

2. Patients beyond the Milan criteria: As a general rule, each single treatment should include a planned dose of up to $150 \mathrm{mg}$ doxorubicin loaded into two vials of DC Beads. In huge or bilobar tumors, treatment typically includes separate sessions $\sim 4$ weeks apart, in the absence of complications that would require a longer time interval between the two sessions. Obtaining confirmation that the liver enzymes have returned to baseline before performing the second treatment session is recommended.

\section{Choice of Embolic Material and Embolization End Point}

In conventional TACE, the administration of the anticancerin-oil emulsion is followed by mechanical embolization with either a spherical or a nonspherical embolic agent. The embolization end point is usually defined as stasis in the second- or third-order branches of the lobar hepatic artery. In DEB-TACE, use of 100 - to $300-\mu \mathrm{m}$ beads is recommended for a standard procedure. This choice is based on the demonstration that such small particles are delivered inside the tumor or in close proximity to the tumor margin, and thus they are ideal for drug delivery or precise embolization. ${ }^{14}$ However, individual patient and tumor characteristics, particularly the identification of arteriovenous shunting, should be taken into account when the safety of the treatment and the choice of bead size are determined. In the case of significant arterioportal or hepatic venous shunting, embolization of the shunt with Gelfoam pledgets or large particles is recommended before proceeding with DEB administration. Angiographic confirmation that the shunt is no longer present must be obtained before DEB injection can be performed, and a larger bead size may be preferred.

Loaded DC Beads should be mixed with a nonionic contrast medium. At least 5 to $10 \mathrm{~mL}$ of nonionic contrast should be used per $1 \mathrm{~mL}$ of DC Bead (i.e., 10 to $20 \mathrm{~mL}$ are required to dilute one vial of DC Bead) prior to injection. A good suspension of DC Beads in the contrast should be ensured before delivery. The injection must be very slow: The authors recommend an injection rate of $1 \mathrm{~mL}$ of the contrast agent/DC Bead suspension per minute. Care should be taken to avoid sedimentation of the beads in the syringe by rotating the syringes or using a threeway stopcock to gently suspend the beads in the solution. ${ }^{14}$

Injection of DEB should be continued until near stasis is observed in the artery directly feeding the tumor (i.e., the contrast column should clear within 2 to 5 heartbeats). At that point, injection must be stopped regardless of the amount of beads that have been actually administered, to avoid reflux of embolic material. Once the embolization end point has been achieved, no additional embolic material should be injected. If the "near stasis" end point is not obtained after injection of the scheduled volume of loaded beads, two different options are possible. One option is to inject additional unloaded beads until the embolization end point has been reached. Another option is not to inject additional unloaded beads and to schedule the patient for a repeat course of treatment as needed after imaging follow-up. There are no sufficient data to mandate one strategy over the other. ${ }^{14}$

\section{Catheter Positioning}

A superselective (i.e., segmental or subsegmental) approach should be used whenever possible by using a microcatheter (-Fig. 2). Use of three-dimensional images obtained from Carm rotational angiography with a flat-panel detector system (cone-beam CT) is recommended, if available, to improve the accuracy in identifying tumor-feeding arteries. ${ }^{15-17}$ In addition, repeat cone-beam CT is recommended after delivery to confirm adequate targeting and saturation of the tumor(s).

\section{Response Assessment}

Assessment of tumor response is of utmost importance in patients undergoing TACE. Unfortunately, conventional methods for response assessment, such as Response Evaluation Criteria in Solid Tumors (RECIST), have no predictive value in HCC patients treated with TACE. ${ }^{18}$ These criteria only rely on tumor shrinkage as a measure of antitumor activity, an assumption that is only valid with cytotoxic drugs. TACE induces direct tumor necrosis, and its anticancer efficacy is not paralleled by a reduction in overall tumor load but rather by a reduction in viable tumor, as identified by contrastenhanced radiologic imaging. A modification of the RECIST criteria, named modified RECIST (mRECIST), for HCC is based

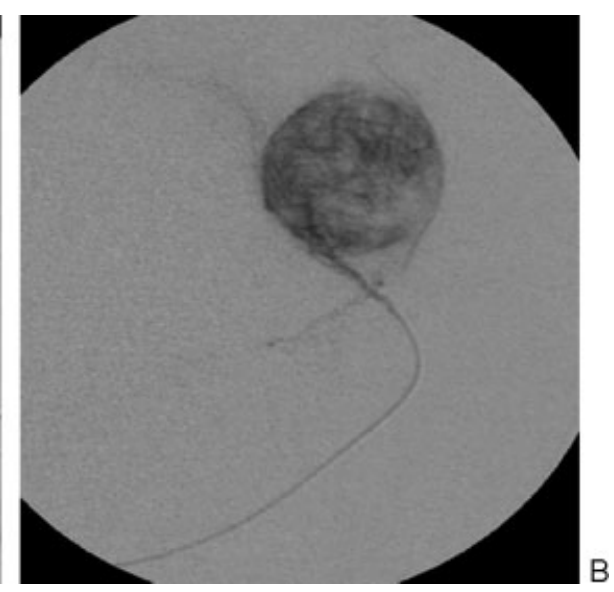

Figure 2 Drug-eluting bead (DEB)-transcatheter arterial chemoembolization. (A) Nonselective angiography and (B) superselective catheterization of the hepatic arterial branch feeding the tumor are performed to administer DEB to the target tumor. 


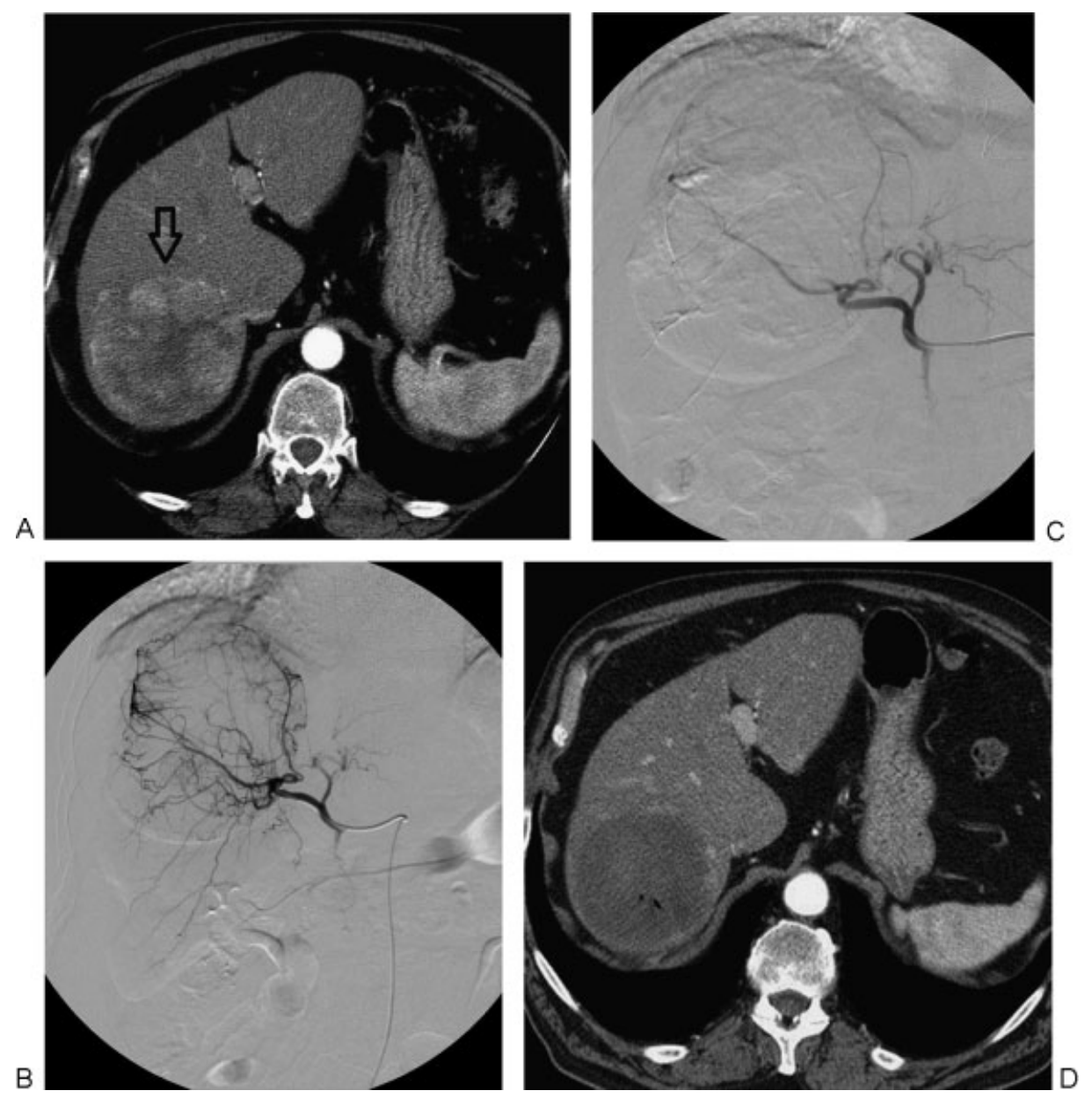

Figure 3 Modified Response Evaluation Criteria in Solid Tumors complete response in a hepatocellular carcinoma (HCC) patient treated with drug-eluting bead (DEB)-transcatheter arterial chemoembolization. (A) Pretreatment arterial-phase computed tomography (CT) shows large hypervascular HCC in segment VII (arrow). Angiograms show (B) catheterization of the hepatic arterial branch feeding the tumor and (C) absence of residual tumor vascularity following DEB administration. (D) CT obtained 1 month after the procedure shows absence of residual viable enhancing tumor tissue.

on the fact that diameter of the target lesions with viable tumor should guide all measurements (-Figs. 3 and $\mathbf{4}$ ). In addition, specific modifications of the original criteria regarding assessment of vascular invasion, lymph nodes, ascites, pleural effusion, and new lesions have been introduced. ${ }^{19}$ Tumor response measured by mRECIST after TACE has been shown to correlate with survival outcomes. ${ }^{20,21}$ The recent Clinical Practice Guidelines jointly issued by the European Association for the Study of the Liver (EASL) and the European Organization for Research and Treatment of Cancer (EORTC) state that assessment of response in HCC should be based on mRECIST criteria by performing contrast-enhanced CT or MRI 4 weeks after initial treatment. ${ }^{7}$

\section{Treatment Schedule}

No randomized trials have been designed to evaluate the optimal frequency of therapy. TACE has been performed both at regular predefined time intervals and "on demand," according to tumor response as observed on imaging. When an "on-demand" strategy is followed, further TACE treatment is usually scheduled in patients with residual viable tumorincluding partial response, stable disease, and progressive disease according to mRECIST- $\sim 8$ weeks after the initial treatment, in the absence of contraindications requiring a longer time interval. Obtaining confirmation that the liver enzymes have returned to baseline before repeating treatment is recommended. In contrast, in patients with no evidence of residual viable disease (i.e., with complete response according to mRECIST), imaging follow-up should be scheduled every 2 to 3 months.

\section{TACE Discontinuation}

TACE should be discontinued, even if technically feasible, in patients presenting with untreatable progression. ${ }^{14}$ Untreatable progression is defined by at least one of the following:

1. Failure to achieve an objective response in the targeted tumor after at least two treatments. Of importance, the emergence of new intrahepatic tumor foci remote from the treated territory, although clearly representing tumor progression according to mRECIST for HCC, does not contraindicate further treatment with TACE.

2. Clinical or functional deterioration. Treatment should be discontinued in patients showing clinical progression to 

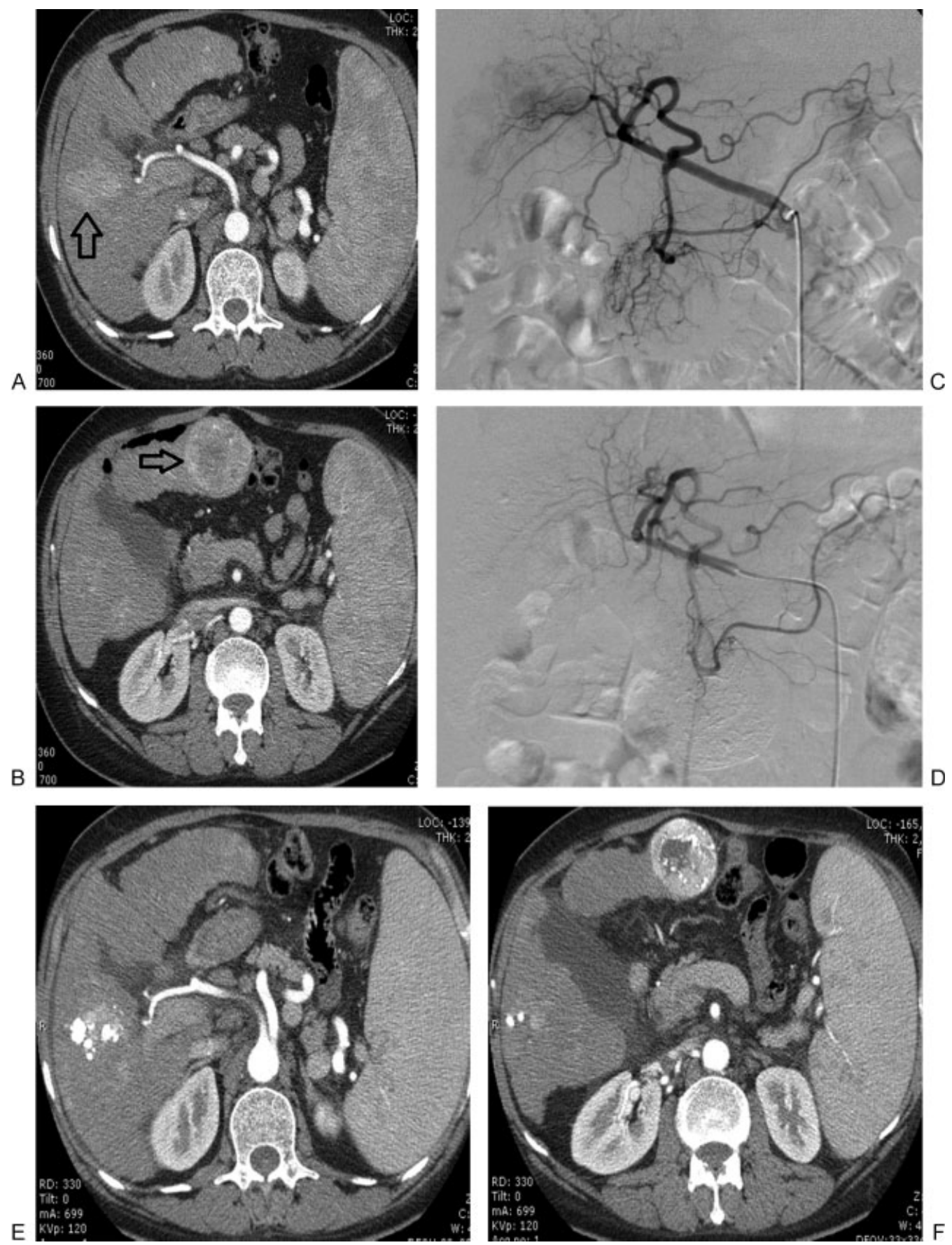

Figure 4 Modified Response Evaluation Criteria in Solid Tumors stable disease in a hepatocellular carcinoma (HCC) patient treated with conventional transcatheter arterial chemoembolization. (A, B) Pretreatment arterial-phase computed tomography (CT) shows multinodular HCC involving both hepatic lobes (arrows). (C, D) After selective catheterization of the right and left hepatic arterial branches, angiography shows elimination of tumor vascularity. (E, F) CT obtained 1 month after the procedure shows persistent active disease with minimal lipiodol retention within the target tumors.

Eastern cooperative Oncology Group (ECOG) performance status $>2$ or evolution to sustained hepatic decompensation (not merely after therapy).

3. Development of a contraindication to TACE therapy once regimens have been initiated.

\section{Indications and Contraindications}

The best candidates for TACE are patients with asymptomatic multinodular tumors without vascular invasion or extrahe- patic spread. ${ }^{7}$ TACE is also offered to patients with early-stage HCC when surgical options or percutaneous ablation are precluded, following the concept of treatment stage migration described in the EASL-EORTC Clinical Practice Guidelines (-Fig. 5). ${ }^{7}$ Liver functional reserve is a critical component for careful patient selection. Patients should present with relatively well-preserved liver function (mostly Child-Pugh A or B7 without ascites); those with liver decompensation or more advanced liver failure should be excluded because the ischemic insult can lead to severe adverse events. -Table 1 

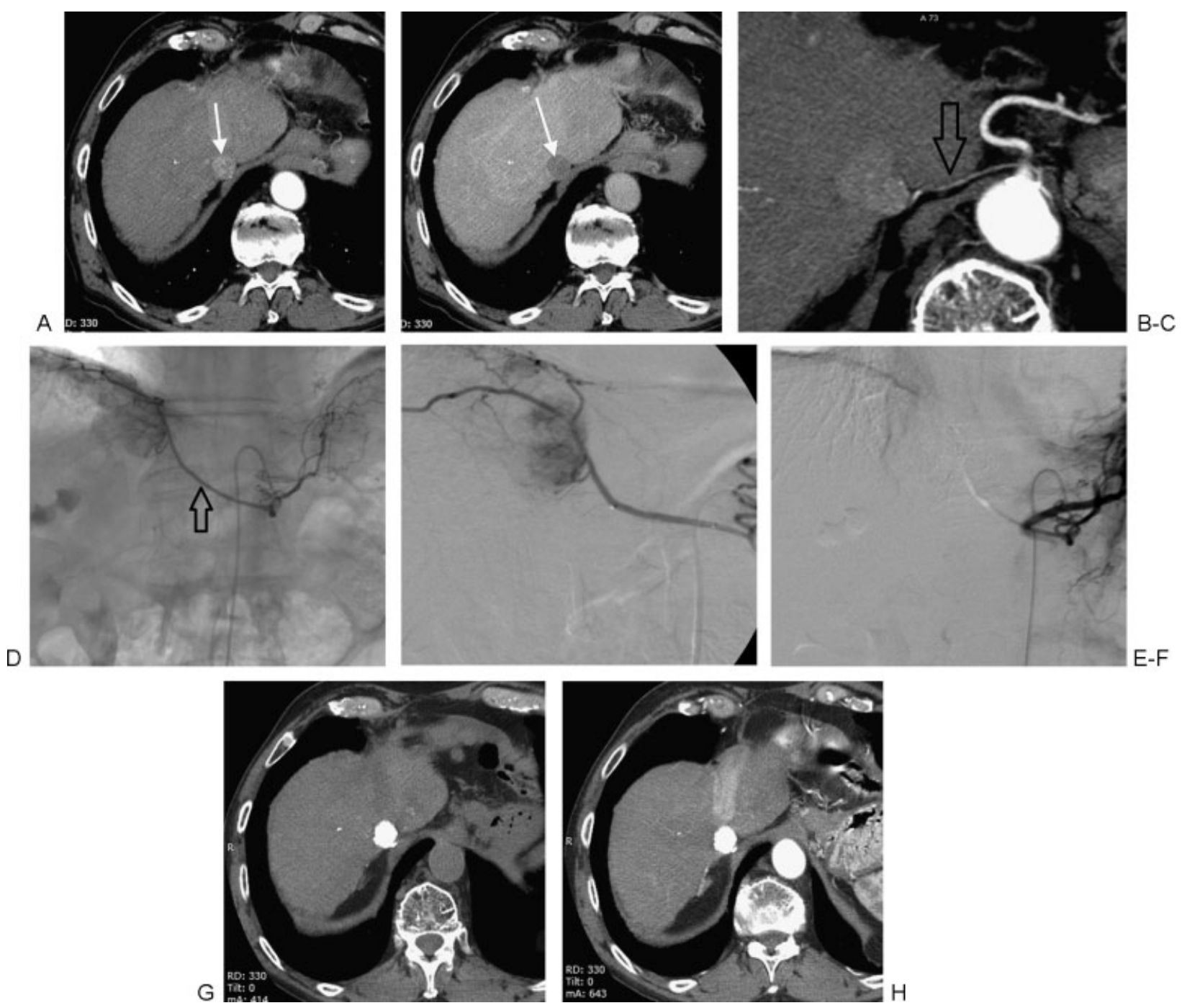

Figure 5 Patient with early-stage hepatocellular carcinoma (HCC) treated with transcatheter arterial chemoembolization following the concept of treatment stage migration. (A, B) Pretreatment arterial-phase computed tomography (CT) shows small solitary HCC (arrows) in a patient who was not a candidate for surgery or percutaneous ablation. (C) CT reformation demonstrates arterial supply to the tumor from the diaphragmatic artery (arrow), confirmed at angiography (D; arrow). (E, F) After superselective catheterization of the right inferior diaphragmatic artery, angiography fails to show residual tumor vascularity. $(\mathrm{G}, \mathrm{H}) \mathrm{CT}$ obtained 1 month after the procedure shows dense and homogeneous lipiodol retention within the target tumor, with no evidence of residual active disease.

summarizes the recommendations for the use of TACE in patients with HCC as reported in the EASL-EORTC Clinical Practice Guidelines. ${ }^{7}$ - Table 2 reports absolute and relative contraindications for the use of conventional TACE in patients with HCC as developed by a multidisciplinary panel of experts. $^{22}$

\section{Clinical Outcomes}

\section{Conventional TACE}

The survival benefit of conventional TACE has been the subject of a limited number of randomized controlled trials that provided contradictory results. ${ }^{13}$ A cumulative metaanalysis of the studies, however, has clearly shown that 2-year survival of patients with HCC not suitable for radical therapies who are treated with arterial embolization or chemoembolization is improved compared with conservative manage- ment. ${ }^{23}$ Sensitivity analysis in this study showed a significant benefit of chemoembolization with cisplatin or doxorubicin assessing by 323 patients in four studies but no benefit with embolization alone by assessing 215 patients in three studies. $^{23}$

In a recent Cochrane meta-analysis, the evidence supporting the benefits of TACE was questioned. ${ }^{24}$ The authors of the Cochrane review state that, contrary to current clinical practice, there is absence of evidence of TACE having a beneficial effect on survival in participants with unresectable HCC. ${ }^{24}$ However, several experts have questioned such conclusions and expressed concern over this Cochrane review. ${ }^{25,26}$ It has been pointed out that the analysis included an randomized controlled trial (RCT) undertaken in patients with early HCC in whom transcatheter arterial embolization (TAE) (not TACE) was assessed in combination with local ablation-and that, on the other hand, it excluded two major trials that found 
Table 1 Recommendations for Transcatheter Arterial Chemoembolization in Patients with Hepatocellular Carcinoma ${ }^{\mathrm{a}}$

- TACE is recommended for patients with intermediate-stage HCC (multinodular asymptomatic tumors without vascular invasion or extrahepatic spread) (GR: 1A)

- TACE is discouraged in patients with decompensated liver disease, advanced liver dysfunction, macroscopic invasion, or extrahepatic spread (GR: 1B)

- The use of drug-eluting beads has shown similar response rates than Gelfoam-lipiodol particles associated with less systemic adverse events (GR: 2B)

- Selective intra-arterial chemotherapy, bland embolization, and lipiodolization are not recommended for the management of HCC (GR: 2B)

Abbreviations: EASL, European Association for the Study of the Liver; EORTC, European Organization for Research and Treatment of Cancer; GR, grade of recommendation; HCC, hepatocellular carcinoma; TACE, transcatheter arterial chemoembolization.

${ }^{a}$ As reported in the Clinical Practice Guidelines jointly issued by the EASL and the EORTC (adapted from reference 7). The recommendations follow the Grading of Recommendations, Assessment, Development, and Evaluation system and are based on the level of evidence and the strength of the data.

improved survival because of the risk of bias according to Cochrane criteria. ${ }^{25,26}$

The outcome of TACE appears to depend on careful patient selection. In a RCT that recruited patients with compensated cirrhosis (70\% in Child-Pugh A), absence of cancer-related symptoms (81\% with ECOG performance status of 0 ), and large or multinodular HCC with neither vascular invasion nor extrahepatic spread, 2-year survival after conventional TACE reached $63 \%$, compared with $27 \%$ of the untreated control arm $(p=0.009) .{ }^{27}$ In contrast, in another RCT, the use of broader

Table 2 Absolute and Relative Contraindications for Conventional Transcatheter Arterial Chemoembolization in Patients with Hepatocellular Carcinoma ${ }^{\mathrm{a}}$

\begin{tabular}{|l|}
\hline Absolute contraindications \\
\hline $\begin{array}{l}\text { Decompensated cirrhosis (Child-Pugh B } 8 \text { or higher) } \\
\text { including: }\end{array}$ \\
\hline - Jaundice \\
\hline - Clinical encephalopathy \\
\hline - Refractory ascites \\
\hline - Hepatorenal syndrome \\
\hline - Extensive tumor with massive replacement of both lobes \\
\hline $\begin{array}{l}\text { - Severely reduced portal vein flow (e.g., nontumoral } \\
\text { portal vein occlusion or hepatofugal blood flow) }\end{array}$ \\
\hline - Technical contraindications to hepatic intra-arterial \\
treatment (e.g., untreatable arterio-venous fistula) \\
\hline - Renal insufficiency (creatinine $\geq 2$ mg/dL or creatinine \\
clearance $\leq 30$ mL/min) \\
\hline Relative contraindications \\
\hline - Tumor size $\geq 10$ cm \\
\hline - Comorbidities involving compromised organ function: \\
\hline - Active cardiovascular disease \\
\hline - Active lung disease \\
\hline - Untreated varices at high risk of bleeding \\
\hline - Bile duct occlusion or incompetent papilla due to stent \\
or surgery
\end{tabular}

Source: Adapted from Raoul et al. ${ }^{22}$

${ }^{\mathrm{a}}$ As developed by a multidisciplinary panel of experts. enrollment criteria with inclusion of patients with symptoms or limited portal vein invasion resulted in a 2-year survival of only $31 \%{ }^{28}$ This figure was still superior to the one of the untreated control group (2-year survival, $11 \% ; p=0.002$ ). In this study, no survival benefit was identified in the subgroup analysis restricted to patients presenting with portal vein invasion. ${ }^{28}$

\section{DEB-TACE}

The clinical value of DEB-TACE has been demonstrated by RCTs. In a multicenter phase 2 randomized trial including 201 European patients (PRECISION V), the use of doxorubicineluting beads resulted in a marked reduction in liver toxicity and drug-related adverse events compared with conventional TACE with doxorubicin. ${ }^{10,11}$ The mean maximum aspartate aminotransferase and alanine aminotransferase increase in the DC Bead group were $50 \%$ and $41 \%$ less than in the conventional TACE group $(p<0.001)$. Owing to the improved safety and tolerability profile, high-dose doxorubicin treatment could be applied according to the planned schedule in the whole drug-eluting bead group, resulting in consistently high rates of objective response and disease control in all subgroup analyses. Contrary to the observation in the DEB arm, the objective response rate (ORR) and the disease control rate (DCR) for conventional TACE in the subgroups of patients with more advanced disease were significantly reduced ( $p=0.038$ for ORR; $p=0.026$ for DCR), although the differences were not statistically significant in the overall patient population (ORR: $52 \%$ versus $44 \%$; DCR: $63 \%$ versus $52 \%$ ). A recent case-control study conducted in Asian patients with HCC confirmed higher ORR for DEB-TACE as compared with conventional TACE. ${ }^{29}$

The added value of chemotherapeutic agent over bland embolic microspheres has been demonstrated by a randomized trial comparing beads loaded with doxorubicin versus bland embolization performed with an embolic microsphere with similar characteristics. The rate of tumor progression at 12 months was significantly lower in the DEB arm than in the bland embolization arm (46\% versus $78 \%, p=0.002$ ), and time to progression (TTP) increased from $36.2 \pm 9.0$ weeks to $42.4 \pm 9.5$ weeks $(p=0.008){ }^{30}$ Another investigation assessed the degree of necrosis in explanted livers after 
chemoembolization with epirubicin-loaded DEB versus bland embolization in patients on a transplant waiting list: DEBTACE achieved complete necrosis in $77 \%$ of lesions, whereas bland embolization achieved complete necrosis in only $27 \%$ of lesions $(p=0.043){ }^{31}$

Despite the absence of phase 3 trials comparing DEB-TACE versus conventional regimens, DEB-TACE has been increasingly used as the first-line transcatheter treatment for HCC. DEB-TACE provides levels of consistency and repeatability not available with conventional TACE, and it offers the opportunity to implement a standardized approach to HCC treatment. A recent publication reported a median survival of 48 months in a cohort of 104 HCC Western patients treated with DEBTACE, a figure that appears to be far superior with respect to those reported for conventional TACE. ${ }^{32}$

\section{Synergies and Combination Strategies}

An important limitation of all TACE regimens is the high rate of tumor recurrence. In RCTs, a sustained response lasting $>3$ to 6 months was observed in only 28 to $35 \%$ of patients who received conventional TACE, and in nonresponders no survival benefit was identified compared with best supportive care. ${ }^{27,28}$ Even in those patients in whom initial response was achieved, the 3-year cumulative rate of intrahepatic recurrence reaches $65 \%$, with recurrent tumor showing significantly shorter median doubling time. ${ }^{33}$ As a result, the 3-year survival rate of TACE-treated patients did not exceed 26 to $29 \%$ in RCTs. ${ }^{27,28}$

Increased understanding of the molecular signaling pathways involved in HCC has led to the development of moleculartargeted therapies aimed at inhibiting tumor cell proliferation and angiogenesis. Sorafenib, a multikinase inhibitor with antiangiogenic and antiproliferative properties, has been shown to prolong median overall survival and median time to radiologic progression compared with placebo in RCTs, and it has become the current standard of care for patients with advanced-stage tumors not suitable for surgical or locoregional therapies. $^{34,35}$

Tumor recurrence following TACE is characterized by increased VEGF production and subsequent angiogenesis. Moreover, TACE increases VEGF expression in the residual surviving cancerous tissue ${ }^{36}$ and induces expression of other proangiogenic factors, such as hypoxia inducible factor $1 \alpha{ }^{37}$ Based on these findings, a combination of TACE with agents with antiangiogenic properties would appear to be a rational approach.

The availability of DEB that minimizes the systemic chemotherapy exposure at the time of TACE is appealing for combination regimens, based on mechanisms that are theoretically synergistic. DEB-TACE has been shown to be safe and effective, with significantly reduced systemic drug exposure compared with conventional TACE regimens. Sorafenib has demonstrated efficacy and safety in patients with advanced HCC, with activity on both tumor cells and endothelial cells. In a prospective single-center phase 2 study, safety and response of a combined protocol involving sorafenib $400 \mathrm{mg}$ twice per day and DEB-TACE were assessed in 35 patients. $^{38}$
Although most patients experienced at least one grade 3 to 4 toxicity, most toxicities were minor (grade 1 to $2,83 \%$ versus grade 3 to $4,17 \%$ ), and preliminary efficacy data were promising.

The phase 2 randomized double-blind placebo-controlled SPACE study (Sorafenib or Placebo in Combination with DEBTACE for Intermediate-Stage HCC) was the first global trial on the use of TACE in the treatment of HCC. ${ }^{39}$ The objective of the study was to evaluate the efficacy and safety of sorafenib in combination with DEB-TACE in patients with intermediatestage HCC. The study was conducted at 85 sites across Europe, North America, and the Asia-Pacific region. Patients were eligible if they had asymptomatic unresectable multinodular tumors without vascular invasion or extrahepatic spread, Child-Pugh A liver functional status, and ECOG performance status $0 .^{39}$ Patients were randomized to receive sorafenib $400 \mathrm{mg}$ twice daily or matching placebo continuously until progression. All patients received DEB-TACE (150 mg doxorubicin) 3 to 7 days after the first dose of the study drug, and then on day 1 ( \pm 4 days) of cycles 3,7 , and 13 , and every six cycles thereafter. Patients were allowed optional DEB-TACE sessions between cycles 7 and 13 and cycles 13 and 19, if deemed necessary by the investigator.

Of 452 patients screened, 307 were randomized to sorafenib $(n=154)$ or placebo $(n=153)$. The study met its primary end point of improving TTP; the TTP for DEB-TACE plus sorafenib was longer than the one for DEB-TACE plus placebo. The hazard ratio for TTP was 0.797 (95\% confidence interval, 0.588 to $1.080 ; p=0.072$; one-sided $\alpha$ : 0.15$)$. The combination was well tolerated, and no new safety findings that would preclude use of the combination were observed. Nevertheless, the encouraging efficacy signal requires confirmation with data from ongoing phase 3 trials. In fact, several questions remain as investigators attempt to improve treatment outcomes in HCC patients. The pathophysiologic complexity of HCC, balanced with a goal of providing effective tumor therapy with preservation of organ function, makes optimal treatment choice a clinical challenge. An understanding of exactly which features of HCC and patient health may predict the clinical outcome of combination regimens is essential for prescribing individualized evidence-based therapeutic strategies. ${ }^{40}$

\section{References}

1 Parkin DM, Bray F, Ferlay J, Pisani P. Global cancer statistics, 2002. CA Cancer J Clin 2005;55(2):74-108

2 Olsen AH, Parkin DM, Sasieni P. Cancer mortality in the United Kingdom: projections to the year 2025. Br J Cancer 2008;99(9): 1549-1554

3 Davis GL, Alter MJ, El-Serag H, Poynard T, Jennings LW. Aging of hepatitis C virus (HCV)-infected persons in the United States: a multiple cohort model of $\mathrm{HCV}$ prevalence and disease progression. Gastroenterology 2010;138(2):513-521, e1-e6

4 Lencioni R. Chemoembolization for hepatocellular carcinoma. Semin Oncol 2012;39(4):503-509

5 Llovet JM, Di Bisceglie AM, Bruix J, et al; Panel of Experts in HCCDesign Clinical Trials. Design and endpoints of clinical trials in 
hepatocellular carcinoma. J Natl Cancer Inst 2008;100(10): 698-711

6 Bruix J, Sherman M; American Association for the Study of Liver Diseases. Management of hepatocellular carcinoma: an update. Hepatology 2011;53(3):1020-1022

7 European Association for the Study of the Liver; European Organisation for Research and Treatment of Cancer. EASL-EORTC clinical practice guidelines: management of hepatocellular carcinoma. J Hepatol 2012;56(4):908-943

8 Lencioni R. Loco-regional treatment of hepatocellular carcinoma. Hepatology 2010;52(2):762-773

9 Varela M, Real MI, Burrel M, et al. Chemoembolization of hepatocellular carcinoma with drug eluting beads: efficacy and doxorubicin pharmacokinetics. J Hepatol 2007;46(3):474-481

10 Lammer J, Malagari K, Vogl T, et al; PRECISION V Investigators. Prospective randomized study of doxorubicin-eluting-bead embolization in the treatment of hepatocellular carcinoma: results of the PRECISION V study. Cardiovasc Intervent Radiol 2010;33(1): 41-52

11 Vogl TJ, Lammer J, Lencioni R, et al. Liver, gastrointestinal and cardiac toxicity in intermediate hepatocellular carcinoma treated with PRECISION TACE with drug-eluting beads: results from the PRECISION V randomized trial. AJR Am J Roentgenol 2011;197(4): W562-W570

12 Lencioni R. Management of hepatocellular carcinoma with transarterial chemoembolization in the era of systemic targeted therapy. Crit Rev Oncol Hematol 2012;83(2):216-224

13 Lencioni R, Crocetti L. Local-regional treatment of hepatocellular carcinoma. Radiology 2012;262(1):43-58

14 Lencioni R, de Baere T, Burrel M, et al. Transcatheter treatment of hepatocellular carcinoma with Doxorubicin-loaded DC Bead (DEBDOX): technical recommendations. Cardiovasc Intervent Radiol 2012;35(5):980-985

15 Kim HC, Chung JW, Park JH, et al. Transcatheter arterial chemoembolization for hepatocellular carcinoma: prospective assessment of the right inferior phrenic artery with C-arm CT. J Vasc Interv Radiol 2009;20(7):888-895

16 Miyayama S, Yamashiro M, Okuda M, et al. Usefulness of conebeam computed tomography during ultraselective transcatheter arterial chemoembolization for small hepatocellular carcinomas that cannot be demonstrated on angiography. Cardiovasc Intervent Radiol 2009;32(2):255-264

17 Miyayama S, Yamashiro M, Hattori Y, et al. Efficacy of cone-beam computed tomography during transcatheter arterial chemoembolization for hepatocellular carcinoma. Jpn J Radiol 2011;29(6): 371-377

18 Forner A, Ayuso C, Varela M, et al. Evaluation of tumor response after locoregional therapies in hepatocellular carcinoma: are Response Evaluation Criteria in Solid Tumors reliable? Cancer 2009;115(3):616-623

19 Lencioni R, Llovet JM. Modified RECIST (mRECIST) assessment for hepatocellular carcinoma. Semin Liver Dis 2010;30(1):52-60

20 Gillmore R, Stuart S, Kirkwood A, et al. EASL and mRECIST responses are independent prognostic factors for survival in hepatocellular cancer patients treated with transarterial embolization. J Hepatol 2011;55(6):1309-1316

21 Shim JH, Lee HC, Kim SO, et al. Which response criteria best help predict survival of patients with hepatocellular carcinoma following chemoembolization? A validation study of old and new models. Radiology 2012;262(2):708-718

22 Raoul JL, Sangro B, Forner A, et al. Evolving strategies for the management of intermediate-stage hepatocellular carcinoma: available evidence and expert opinion on the use of transarterial chemoembolization. Cancer Treat Rev 2011;37(3):212220

23 Llovet JM, Bruix J. Systematic review of randomized trials for unresectable hepatocellular carcinoma: chemoembolization improves survival. Hepatology 2003;37(2):429-442
24 Oliveri RS, Wetterslev J, Gluud C. Transarterial (chemo)embolisation for unresectable hepatocellular carcinoma. Cochrane Database Syst Rev 2011;3(3):CD004787

25 Ray CE Jr, Haskal ZJ, Geschwind JFH, Funaki BS. The use of transarterial chemoembolization in the treatment of unresectable hepatocellular carcinoma: a response to the Cochrane Collaboration review of 2011. J Vasc Interv Radiol 2011;22(12):1693-1696

26 Forner A, Llovet JM, Bruix J. Chemoembolization for intermediate HCC: is there proof of survival benefit? J Hepatol 2012;56(4): 984-986

27 Llovet JM, Real MI, Montaña X, et al; Barcelona Liver Cancer Group . Arterial embolisation or chemoembolisation versus symptomatic treatment in patients with unresectable hepatocellular carcinoma: a randomised controlled trial. Lancet 2002;359(9319): $1734-1739$

28 Lo CM, Ngan H, Tso WK, et al. Randomized controlled trial of transarterial lipiodol chemoembolization for unresectable hepatocellular carcinoma. Hepatology 2002;35(5):1164-1171

29 Song MJ, Park CH, Kim JD, et al. Drug-eluting bead loaded with doxorubicin versus conventional lipiodol-based transarterial chemoembolization in the treatment of hepatocellular carcinoma: a case-control study of Asian patients. Eur J Gastroenterol Hepatol 2011;23(6):521-527

30 Malagari K, Pomoni M, Kelekis A, et al. Prospective randomized comparison of chemoembolization with doxorubicin-eluting beads and bland embolization with BeadBlock for hepatocellular carcinoma. Cardiovasc Intervent Radiol 2010;33(3):541-551

31 Nicolini A, Martinetti L, Crespi S, Maggioni M, Sangiovanni A. Transarterial chemoembolization with epirubicin-eluting beads versus transarterial embolization before liver transplantation for hepatocellular carcinoma. J Vasc Interv Radiol 2010;21(3): 327-332

32 Burrel M, Reig M, Forner A, et al. Survival of patients with hepatocellular carcinoma treated by transarterial chemoembolisation (TACE) using Drug Eluting Beads. Implications for clinical practice and trial design. J Hepatol 2012;56(6):1330-1335

33 Woo HY, Jang JW, Choi JY, et al. Tumor doubling time after initial response to transarterial chemoembolization in patients with hepatocellular carcinoma. Scand J Gastroenterol 2010;45(3): 332-339

34 Llovet JM, Ricci S, Mazzaferro V, et al; SHARP Investigators Study Group. Sorafenib in advanced hepatocellular carcinoma. N Engl J Med 2008;359(4):378-390

35 Cheng AL, Kang YK, Chen Z, et al. Efficacy and safety of sorafenib in patients in the Asia-Pacific region with advanced hepatocellular carcinoma: a phase III randomised, double-blind, placebocontrolled trial. Lancet Oncol 2009;10(1):25-34

36 Wang B, Xu H, Gao ZQ, Ning HF, Sun YQ Cao GW. Increased expression of vascular endothelial growth factor in hepatocellular carcinoma after transcatheter arterial chemoembolization. Acta Radiol 2008;49(5):523-529

37 Virmani S, Rhee TK, Ryu RK, et al. Comparison of hypoxia-inducible factor-1alpha expression before and after transcatheter arterial embolization in rabbit VX2 liver tumors. J Vasc Interv Radiol 2008;19(10):1483-1489

38 Pawlik TM, Reyes DK, Cosgrove D, Kamel IR, Bhagat N, Geschwind JF. Phase II trial of sorafenib combined with concurrent transarterial chemoembolization with drug-eluting beads for hepatocellular carcinoma. J Clin Oncol 2011;29(30):3960-3967

39 Lencioni R, Llovet JM, Han G, et al. Sorafenib or placebo in combination with transarterial chemoembolization (TACE) with doxorubicin-eluting beads (DEBDOX) for intermediate-stage hepatocellular carcinoma (HCC): phase II, randomized, double-blind SPACE trial. [abstract LBA154]. J Clin Oncol 2012;30(Suppl 4):

40 Gish RG, Lencioni R, Di Bisceglie AM, Raoul JL, Mazzaferro V. Role of the multidisciplinary team in the diagnosis and treatment of hepatocellular carcinoma. Expert Rev Gastroenterol Hepatol 2012;6(2):173-185 